\title{
SAXS: A Versatile Tool to Study Biological Macromolecules in Solution
}

The Biophysics Collaborative Access Team (BioCAT), which operates the high-brilliance undulator Beamline 18ID at the Advanced Photon Source, Argonne National Laboratory, is dedicated to studying biological macromolecules in solution using Small-Angle X-ray Scattering (SAXS). The combination of a highly brilliant X-ray source and advanced micro-focusing optics at BioCAT enables significant insights into the structure and dynamics of bio-macromolecules from both equilibrium and time-resolved SAXS experiments.

The quality and interpretability of SAXS data is largely contingent on sample homogeneity. Size-exclusion chromatography (SEC)-SAXS, which was pioneered at BioCAT has therefore emerged as a powerful mode of data acquisition as it ensures optimal sample quality by purification immediately before exposure to x-rays. In addition, we have also incorporated multi-angle light scattering (MALS), dynamic light scattering (DLS), and differential refractive index (dRI) detectors in line with the SAXS camera thus enabling acquisition of a full complement of biophysical data typically required to structurally characterize biological macromolecules simultaneously with SAXS data.

The new frontier in the field is to study real-time structural changes in biological systems as a response to very specific environmental stimuli such as a change in denaturant concentration, ionic strength, $\mathrm{pH}$ or ligand binding. Forefront time resolved SAXS techniques using chaotic mixing continuous flow, continuous laminar flow and stopped flow mixing are now available to a wide variety of bio-medical projects at BioCAT primarily on account of being optimized over the past few years both for sample consumption and mixing efficiency.

Srinivas Chakravarthy Thomas Irving Osman Bilsel

Sagar Kathuria
Illinois Institute of Technology Illinois Institute of Technology University of Massachusetts, Medical School University of Massachusetts, Medical School 